\title{
La singularidad según la educación personalizada en la era digital
}

\author{
PAOLA PEROCHENA GONZÁLEZ* \\ GISELA MATILDE CORIA ${ }^{* *}$ \\ JOSÉ FERNANDO CALDERERO HERNÁNDEZ \\ Universidad Internacional de La Rioja - España
}

Recibido el 05-03-2016; primera evaluación el 28-04-2016; segunda evaluación el 09-06-2016; aceptado el 13-07-2016

\begin{abstract}
* Doctora por la Universidad de Salamanca. Máster TIC en Educación por la misma Universidad y máster en Humanidades por la Universidad Francisco de Vitoria. Licenciada en Psicología por la Universidad Iberoamericana. Actualmente es profesora de Didáctica General (y otras asignaturas) en la Universidad Internacional de La Rioja (UNIR) para los Grados en Magisterio Primaria e Infantil y de Máster en Formación del Profesorado de Secundaria y ha sido coordinadora del Departamento de Psicopedagogía de la Facultad de Educación de esta Universidad. Miembro del Grupo de Investigación «Educación Personalizada en la Era Digital (EPEDIG)». Ha participado y coordinado procesos de enseñanza-aprendizaje a distintos niveles (formación reglada y no reglada, formación del profesorado y en la empresa). Tiene publicaciones en congresos, libros y en revistas científicas. Ha colaborado en diversos proyectos de investigación con financiación privada y pública por el Gobierno de España. Es miembro de asociaciones dedicadas a la investigación en el ámbito universitario. Tiene experiencia en el diseño y elaboración de materiales multimedia y gestión de plataformas de formación así como en la organización de congresos académicos. Contacto: paola.perochena@unir.net

** Doctora por la Universidad de Zaragoza, ha realizado el programa de Doctorado «Psicología y Aprendizaje» dirigido a profundizar en los procesos de aprendizaje tanto en ámbitos formales como no formales. Licenciada en Psicología por la Universidad de Rosario (título homologado en España) con formación en el ámbito clínico, educativo, jurídico, laboral y social. Ambas formaciones junto a la participación en proyectos socioeducativos han orientado sus intereses hacia el área de conocimiento Psicología Evolutiva y de la Educación. Ha trabajado como profesora en los Grados de Maestro en Educación Primaria e Infantil y como directora de Trabajo Fin de Grado. Desde hace unos años es investigadora en el Grupo de Investigación EPEDIG «Educación Personalizada en la Era Digital» de la Universidad Internacional de La Rioja. Sus inquietudes investigadoras giran en torno a tres líneas: La atención a la diversidad en el ámbito educativo, La singularidad en la educación personalizada en contextos diversos, El desarrollo humano y sus implicaciones escolares. Contacto: gisela.matilde@unir.net

*** Doctor en Filosofía y Ciencias de la Educación por la Universidad Complutense de Madrid. Licenciado en Ciencias Químicas por la Universidad de Salamanca. Profesor de Teoría y Práctica de la Investigación Educativa en la Universidad Internacional de La Rioja, miembro del Grupo de Investigación Educación Personalizada en la Era Digital (GdI-EDUC-04_EPEDIG), director del Seminario Internacional de Educación Personalizada (SIEP), presidente del Capítulo de Educación de AEDOS, asociación para el estudio de la doctrina social de la Iglesia católica, vicepresidente de la Fundación Padres por la Excelencia, Padrex, autor de libros educativos y director de tesis doctorales. Contacto: josefernando.calderero@unir.net
\end{abstract}




\section{RESUMEN}

La singularidad, la apertura y la autonomía son elementos esenciales de la persona considerados fundamentales en la "educación personalizada». El artículo profundiza en las consecuencias de la singularidad en la práctica cotidiana de la enseńanza y el aprendizaje en el contexto actual caracterizado, entre otros aspectos, por una creciente digitalización. Se concluye que el ser único e irrepetible de la persona debe ser considerado en relación con la realidad docente y educativa desde la planificación, ejecución y evaluación. Todo proceso formativo ha de adaptarse a cada situación concreta y a las características e intereses del estudiante, fomentando su capacidad investigadora y su creatividad. El docente adopta, entre otros, un rol de aprendiz y la evaluación se convierte en una fuente de aprendizaje, mejorando así la calidad de la formación.

Palabras clave: educación personalizada, teorías de la educación, singularidad, creatividad.

\section{Singularity According to Personalized Education in Digital Era}

\section{Abstract}

Singularity, opening and autonomy are essential elements considered fundamental in «Personalized Education». The article delves into the consequences of the singularity of every day practice of teaching and learning in the current context characterized, among other features, by increasing digitization. In the light of the outcome, it follows that the unique and unreapetable being of the person must be considered in relation to the teaching and educational reality from planning, execution and assessment. Every training process has to adapt to each specific situation and the features and interests of the student encouraging his/her research capacity and creativity. The teacher adopts, among others, a role of trainee and assessment becomes a source of learning, improving in this way the quality of academic training.

Keywords: customized education, educational theories, singularity, creativity.

\section{A singularidade na perspectiva da educação personalizada na era digital}

\section{Resumo}

A singularidade, a abertura e a autonomia são elementos essenciais da pessoa e fundamentais na «educação personalizada». O artigo aprofunda as consequencias da singularidade na prática cotidiana do ensino e da aprendizagem no contexto atual caracterizando-se, entre outras coisas, por meio do aumento da digitalizaçáo. Conclui-se que o ser único e irrepetível da pessoa deve ser considerado na relação com a realidade docente e educativa desde o planejamento, a execução e a avaliação. Todo o processo formativo deve se adaptar a cada situação concreta e às características e interesses do estudante, estimulando a sua capacidade investigativa e criativa. 
O docente adota, entre outras questóes, um papel de aprendiz e a avaliação se torna uma fonte de aprendizagem, melhorando assim a qualidade da formação.

Palavras chave: educação personalizada, teorias da educação, singularidade, criatividade.

\section{Presentación y contexto}

Desde la publicación del Tratado de Educación Personalizada (coordinado por Víctor García Hoz) tres ejes esenciales dan entidad y operatividad a esta concepción educativa: singularidad, autonomía y apertura (Palacios et al., 1989; García Hoz, 1993). Este ensayo se centra en el eje de la singularidad y forma parte de una línea de investigación del Grupo de Investigación «Educación Personalizada en la Era Digital» (EPEDIG) ${ }^{1}$. Una de las finalidades del Grupo es la profundización en los conceptos fundamentales de la educación personalizada y en sus consecuencias operativas de forma que puedan informar el ejercicio de la actividad docente y educativa.

En la actualidad, tanto investigaciones y metodologías didácticas como normativas legales ponen énfasis en que el estudiante debiera ser el centro de la educación, de la enseñanza-aprendizaje (Calderero, Aguirre, Castellanos, Peris $\&$ Perochena, 2014). Parece necesario subrayar que el principal protagonista, el estudiante, es una persona. La educación personalizada se centra en el carácter personal del ser humano profundizando, desde esta perspectiva, en el diseño, implementación y valoración de la enseńanza-aprendizaje de forma operativa.

En este artículo pretendemos mostrar cómo todo proceso educativo ha de tener muy en cuenta la singularidad de cada persona. Incidimos en algunas de las relaciones conceptuales y operativas de la inherente e innata creatividad de todo ser humano con su singularidad. También nos planteamos la necesidad de reconsiderar los fines y la implementación de los procesos de planificación, ejecución y evaluación, de modo que tengan en cuenta y fomenten la singularidad de cada alumno. Por último, presentamos algunos criterios que ayuden a diseñar e implementar estos procesos. Nuestro planteamiento de fondo en este ensayo como Grupo de Investigación tiene como punto de referencia la necesidad de tener muy en cuenta las características de nuestra época, que bien podríamos denominar «era digital»; por ello consideramos que todos los conceptos trabajados a lo largo del texto deben entenderse en plena conexión con esta denominación, cuyo alcance configura incluso aspectos importantes de la vida humana.

1 EPEDIG pertenece a la Universidad Internacional de La Rioja (UNIR), España, cuya actividad es parcialmente financiada por UNIR Research (http://research.unir.net), dentro del Plan Propio de Investigación, Desarrollo e Innovación. 


\section{LA EDUCACIÓN PERSONALIZADA Y LAS NOTAS CARACTERÍSTICAS DE LA PERSONA}

La educación personalizada entiende que el centro del proceso educativo es el alumno y que existen tres principios insoslayables característicos de la persona: la autonomía, la apertura y la singularidad (García Hoz, 1988). Para lograr una verdadera educación, se hace necesario que el educador reconozca y estimule estos tres aspectos del educando. Aunque estos elementos se separen conceptualmente para ser estudiados, se interrelacionan intrínsecamente, por lo que se hace necesaria una visión global e integral de la persona (Burgos, 2007). Según Maritain (1947) es necesaria una visión de unidad en el ser de los elementos que constituyen esencialmente al ser humano. Por su parte, Jenkins y Keefe (2001) insisten en que la educación debe centrarse en las necesidades e intereses de cada uno de los estudiantes que deben ser atendidos a través de las acciones formativas. Dichas acciones no deben ser consideradas de forma aislada sino con una visión unitaria que aporte coherencia al proceso educativo.

\section{LA SINGULARIDAD}

\subsection{Nota esencial de la persona}

Si bien las personas humanas tenemos la misma naturaleza, una naturaleza humana, cada una es distinta a las demás. Esta singularidad supone trabajar (aunque no solo) desde las diferencias que existen entre las personas, puesto que cada persona es única e irrepetible, insustituible e inintercambiable (Artamendi, 2007); es diversa: «di-versa», es — por decirlo así- una «versión» del ser persona.

En la década de 1980 en el ámbito escolar, la atención a la diversidad y la noción de necesidades educativas especiales (NEE) dieron lugar a una nueva etapa más inclusiva en las propuestas escolares, tanto organizativas y curriculares. En la actualidad, el concepto necesidades educativas personales (NEP) (García, 2013), que va más allá de las NEE, se basa en la premisa de que todos somos diferentes por lo que todos y cada uno tenemos nuestras propias necesidades educativas personales y singulares a las que se debe dar respuesta a través de los procesos formativos.

Desde la ontología, el ser de cada persona humana supone una distinción tanto cualitativa como cuantitativa puesto que cada uno es como es y es distinto de los demás. Es singular y única, tiene su propia e irrepetible identidad (Palacios et al., 1989). Incluso — no siendo el único argumento- desde los estudios 
actuales del genoma se ha demostrado este carácter singular de cada persona humana dado que posee un código genético que se establece en la concepción y que se conserva a lo largo de toda la vida (Jouve, 2013). Palacios et al. (1989) explica que la persona está en constante búsqueda y en un esfuerzo continuo de personalización; en un permanente avance y conquista de su «sí mismo». Ese concepto "sí mismo" aparece repetidas veces en su obra haciendo referencia a aquello que no cambia pero que se va descubriendo y que estaría imbricado también en la apertura y la originación de las que habla Bernardo (2011a).

Cabe resaltar que cada persona tiene un modo de ser que es solo suyo, que no le pertenece a nadie más y que solo él o ella es capaz de desarrollar, su identidad personal: cada uno es idéntico solo a sí mismo. El filósofo personalista Zubiri sería quien acuñó este concepto de sí mismo. Finalmente, de la singularidad se desprende el sentido de responsabilidad hacia el propio aprendizaje porque es la vida interior (el propio yo) el que se va desarrollando de modo singular y único que, por supuesto, está en estrecha relación con las circunstancias externas (Bernardo \& Javaloyes, 2015).

\subsection{Creatividad y singularidad}

La persona es creativa por su propia naturaleza. Por ello y, desde el ámbito educativo, García Hoz (1988) identifica la creatividad como un aspecto destacable de la singularidad del ser personal, sostiene que la capacidad creativa es un principio unificador del proceso formativo y que el hacer y pensar creativos se nutren de aspectos emocionales y cognitivos, ofreciendo nuevas soluciones y evitando la despersonalización y la masificación. De hecho, en relación con la singularidad, la educación personalizada supone el cultivo de la intimidad y de la creatividad: la intimidad implica volverse sobre sí mismo y «reflexionar-se»; mientras que la creatividad se encuentra estrechamente ligada a la originalidad, en el sentido de ser capaz de ser origen de algo nuevo mediante aportaciones inéditas, singulares. De este modo, la actividad creadora es el motor del progreso personal y social, y se considera el quehacer más propio y completo de esta concepción educativa (Bernardo, 2007).

La personalización de la educación implica reconocer la originalidad y potenciar las habilidades creadoras. Si bien, la creatividad es una condición inherente a todo ser humano, gracias a la experiencia y al conocimiento podemos construir productos creativos y ofrecer nuevas soluciones a los problemas que se nos presentan (Rendón, 2003). La creatividad también está influenciada por aspectos individuales (incluidos los genéticos), por la calidad de las experiencias educativas y por la estimulación social. 
Por otra parte, desde el constructivismo de Piaget se puede deducir que el conocimiento es una reelaboración que hace el aprendiz, una creación que conjuga los nuevos saberes con las ideas previas. El pensamiento lateral es una forma específica de organizar los sistemas de pensamiento que posibilita la obtención de ideas innovadoras y que se nutre de la creatividad. Ante un problema, no solo se contemplan las posibles opciones lógicas de solución, sino que se proponen nuevas alternativas que no forman parte del pensamiento habitual, que no siguen patrones conocidos, pero que permiten la resolución del problema de manera indirecta y creativa. De esta manera, surgirían ideas ingeniosas y diferentes. (De Bono, 2012). En el ámbito educativo, despertar la curiosidad y el interés constituye el punto de partida de producciones y expresiones creativas, como se verá más adelante.

\section{Algunos aspectos de la SOCIEDAd aCtUAL EN SU RELACión CON LA SINGULARIDAD DE CADA PERSONA Y LA DIGITALIZACIÓN}

Sin pretender agotar el tema, exponemos algunas características de la sociedad actual más relacionadas con el ámbito educativo. Teniendo en cuenta que en la actualidad en los ámbitos educativos hay una evidente proliferación de recursos tecnológicos de naturaleza digital no tendría sentido organizar los procesos de enseñanza-aprendizaje sin tener en cuenta esta realidad contextual y operativa. Nuestro equipo de trabajo se sitúa en un punto medio entre la consideración de asociar toda innovación educativa casi exclusivamente al uso de medios digitales y resaltar excesivamente los peligros que pueden conllevar. Podíamos resumir nuestra posición diciendo que hoy día el trabajo docente no puede hacerse ni sin TIC ni solo con TIC (Velasco, 2015).

\subsection{De consumidores de información a productores de ideas}

Es evidente que en la actual era digital existe un creciente uso de las denominadas tecnologías de la información y la comunicación (TIC) que, sin lugar a dudas, han modificado la naturaleza del trabajo y la organización de la producción. La transformación de la sociedad industrial en la sociedad informacional se caracteriza por el surgimiento de un nuevo paradigma histórico, político, económico, tecnológico y simbólico; la generación, el almacenamiento y la distribución de la información ocupan un lugar central llegando a alcanzar prácticamente todos los ámbitos de la vida de las personas, como apunta Castells (1997).

Es posible acceder a muy diversa información de forma inmediata, lo que hace más necesario desarrollar la capacidad de priorizar, distinguiendo 
lo fundamental, importante, esencial, trascendente, transformando la información en conocimiento significativo. Esto implicaría poseer habilidades específicas, lo cual requiere un giro en la formación y preparación del docente y del discente para que pasen de ser meros consumidores de información a productores de conocimiento (Gros, 2009). El acceso inmediato a la información no necesariamente significa que esta sea la más importante; de ahí que, para una mejor gestión del tiempo convenga distinguir entre lo urgente y lo importante (Paul \& Stroh, 2006).

Coincidimos con Meléndez (2014) en que las aplicaciones digitales favorecen el desarrollo de la singularidad y otras dimensiones de la persona humana y que mal usadas pueden ser un instrumento despersonalizador convirtiendo a las personas en meros consumidores de productos y servicios no siempre beneficiosos.

\subsection{Identidad personal, identidad pública e identidad digital}

El surgimiento de la escuela moderna tiene como finalidad cierta homogenización de la cultura. En este sentido, Vila y Casares (2009) estiman que en la educación formal, el desarrollo de la singularidad sigue siendo una materia pendiente. La educación personalizada considera la identidad de cada persona en cuanto ser singular, para que, siendo ella misma, pueda vivir como parte de un grupo.

En un estudio reciente de Bernal (2014) parece haberse demostrado que los adolescentes con una identidad personal mejor lograda tienen mayor espíritu emprendedor. En dicho estudio se consideró la identidad como aquel elemento que supone la singularidad pero al mismo tiempo la pertenencia a determinados grupos. Como se ha indicado previamente, el uso de las tecnologías cada vez configura más nuestro modo de vivir y el modo de relacionarnos (Area \& Ortíz, 2000). Así, las personas vamos mostrando lo que podríamos denominar una «identidad digital» en la que exteriorizamos aquello de nosotros mismos que será público (Serrano, 2013).

\subsection{Educar en la incertidumbre y la complejidad}

Escotet (2002) describe las características propias del nuevo siglo; entre ellas, la composición multicultural de las sociedades, las estructuras de comunicación de la información, el empleo cotidiano de las tecnologías, las nuevas dimensiones del trabajo (capacidad de iniciativa personal y colectiva, interdisciplinariedad, movilidad de los perfiles profesionales) y la sustitución del Estado-nación por superestructuras económicas y sociales. Estos aspectos dan 
lugar a lo que el autor denomina el siglo de la incertidumbre y la complejidad. El mundo plural, diverso y multicultural requiere de personas que sean capaces de comprender esta realidad incierta y cambiante, pudiendo establecer vínculos valiosos con ella (Calderero et al., 2014) e incluso transformarla.

Por su parte, De Bono (2012) plantea que el pensamiento tradicional, basado en la discusión y la argumentación lógica, recrea situaciones estándares a partir del pasado y las aplica al presente, cuestión que parece funcionar bien en un mundo estable. Sin embargo, en el contexto actual, este sistema de pensamiento no constructivo es insuficiente. «En lugar de juzgar nuestro camino hacia adelante, necesitamos diseñar ese camino. Tenemos que pensar en lo que puede ser, no solo en lo que es» (p. 20).

Campos (2008) argumenta que ante a la realidad incierta y compleja en la que nos desenvolvemos, la educación ha de favorecer la generación de estrategias para la vida. «Educar para la vida es educar para la incertidumbre y el cambio permanentes, es una actitud de sobrevivencia lo que se necesita desarrollar en nuestra población estudiantil» (p. 11).

«En el marco de incertidumbre y de contingencia que caracteriza nuestra existencia, la responsabilidad por uno mismo implica tomar las riendas de nuestra propia vida» (Bernal, 2012 p. 78); precisamente en esa responsabilidad por la propia vida se descubren potencialidades singulares a pesar de que aparentemente se vive en una sociedad con falta de certezas como indican Martín, Novella y Puig (2013).

\section{LA SINGULARIDAD EN LAS TRES ETAPAS DE LOS PROCESOS FORMATIVOS}

De forma sucinta, un proceso formativo tiene tres etapas: planificación, ejecución y evaluación. La planificación sería el núcleo del proceso donde se eligen las metodologías y recursos para conseguir los objetivos, los resultados, las competencias que se pretenden. En la puesta en marcha, el rol del estudiante y del profesor se ejercen en una interacción continua. Una de las funciones de la evaluación es conseguir datos para tomar decisiones respecto al aprendizaje, lo que requiere generar evidencias. A través del aprendizaje que los estudiantes obtienen se podrá valorar si la acción formativa ha conseguido los resultados que pretendía; de ahí que esa información sirva para alimentar la planificación. En cada uno de los tres momentos se requiere esencialmente considerar la singularidad de la persona, tanto del docente como del estudiante. A continuación se expresan algunos aspectos operativos que se derivan de ello en la era digital en la que nos encontramos. 


\subsection{Planificación de la enseñanza desde la singularidad}

Desde la singularidad, en la planificación de la enseñanza-aprendizaje, conocer a cada estudiante se convierte en pieza clave pues será precisamente ese conocimiento el que permita no tanto «adaptar» sino diseñar procesos para atender a las necesidades de cada uno (García, 2013) y especialmente servirá para entablar una relación, que se convierta en un encuentro interpersonal, teniendo en cuenta que la didáctica estudia el encuentro entre profesor y alumno para el aprendizaje (Pariente \& Perochena, 2013), encuentro que también ocurre entre alumnos.

La planificación basada en el carácter personal del ser humano ha de centrarse en la apertura, la autonomía y la expresión del ser singular. Una práctica educativa coherente con la naturaleza humana tiene que partir de los intereses, deseos, inquietudes y necesidades personales. Tonucci (2008) sostiene que la misión de la escuela ya no es «enseñar cosas» pues los estudiantes acceden fácilmente a la información por diversos medios, la planificación debería partir de los intereses y experiencias particulares de los estudiantes para aprender a utilizar esas herramientas y desarrollar métodos de trabajo e investigación que fomenten el conocimiento crítico y las aportaciones singulares al trabajo colaborativo.

En los procesos educativos se planifica a partir de objetivos y finalidades que deberían contemplar las distintas dimensiones de la persona. Para poder trabajar desde esta complejidad, García Hoz (1988) diferencia entre objetivos comunes e individuales. Los primeros serían obligatorios e indispensables para que se establezca la comunicación y la cohesión en el grupo y para atender a la función certificadora de la evaluación; los segundos serían objetivos libres que nacen de la iniciativa y motivación del alumno con orientación del docente. Ahora bien, una clave importante en el proceso de planificación es identificar la forma en la que se evidenciará que dichos objetivos han sido alcanzados. Esto significa que el diseńo de la evaluación debe conformar uno de los primeros pasos en la planificación.

Dentro de la planificación de la enseñanza, una acción esencial es la selección de los medios y recursos a utilizar dependiendo de las funciones que desempeñen (Marquès, 2000). Es relevante contar con las posibilidades reales del centro y con la potencialidad creadora de alumnos y profesores en el diseńo y confección de nuevos medios didácticos. Desde el punto de vista de Bravo (2004), por ejemplo, algunos de ellos podrían utilizarse como refuerzo de la acción del profesor en momentos donde la actividad sea más expositiva (y, por tanto, receptiva de los estudiantes), otros que, siendo autosuficientes en 
la explicación de su contenido, pueden permitir la autonomía en el estudio (como pueden ser videotutoriales) y un tercer ejemplo serían aquellos que facilitan la comunicación entre los implicados en la enseñanza-aprendizaje. Es un reto docente actual el seleccionar el soporte digital o no digital con el que se lleven a cabo estas acciones dentro del proceso.

Una propuesta para la generación de contextos creativos de aprendizaje está basada en la observación de que en la vida cotidiana, en el hogar, la escuela, la sociedad, nos encontramos con diversos objetos, situaciones y personas que nos permiten desarrollar nuestras potencialidades creadoras. En el contexto del aula, la incorporación de objetos y situaciones extrañas y descontextualizadas aportan también múltiples ocasiones para construir nuevo conocimiento. La incertidumbre característica de las situaciones descontextualizadas estimula la fantasía, la curiosidad y el surgimiento de interpretaciones y comprensiones nuevas, además, promueven el aprendizaje colaborativo y la autorregulación de los procesos (Elisondo, Rinaudo \& Donolo, 2011). Por tanto, en la fase de planificación se hace necesario un cierto margen para la espontaneidad de modo que exista la posibilidad de generar sorpresa y expectación en el estudiante que a su vez fomentará la generación de nuevas ideas.

Según Elisondo et al. (2011), si entendemos la creatividad como un proceso complejo en el que intervienen condicionantes cognitivos, motivacionales, afectivos y situacionales, la práctica escolar consistiría en habilitar espacios educativos creativos que atiendan a la totalidad de las variables desde una perspectiva global.

La enseñanza y aprendizaje desde el punto de vista de la adquisición del conocimiento asemeja el papel del alumno al de un investigador. Planificando así la enseñanza, se parte de preguntas o hipótesis de trabajo sobre una problemática para realizar un proceso personal de búsqueda y desarrollo explicativo de la situación. El potencial de la formulación de problemas radica en la posibilidad de que cada alumno pueda crear puentes intra e interdisciplinares y puentes entre los contenidos académicos y su vida cotidiana.

\subsection{Ejecución de la enseñanza y aprendizaje desde la singularidad}

En todo proceso de enseñanza-aprendizaje existen dos roles fundamentales, educador y educando, donde cada uno ejerce un papel distinto pero comparten el mismo fin que fundamentalmente es el aprendizaje del segundo; para ello, no es suficiente planificar sino que la ejecución ha de ser flexible.

La consecuencia lógica de la singularidad en la ejecución de los procesos formativos sería que la atención a cada uno debiera ser individual y, si bien es 
cierto que una tutoría (mentoría, coaching o preceptuación) que atienda uno a uno a los estudiantes puede favorecer el crecimiento de ellos (Wisker, Exley, Antoniou \& Ridley, 2012), no es esta la única seña de identidad de la educación personalizada. De hecho, puesto que cada estudiante es único, también será único lo que pueda aportar a un grupo, por ejemplo, cuando se trabaja en equipo en clase. Igualmente, por la relación entre la singularidad y la apertura, la persona es profundamente creativa, capaz de producir ideas (o cosas) originales, de transformar la realidad y de reflexionar sobre sí mismo, como hemos indicado con anterioridad y que se pone en juego durante la ejecución.

García Hoz (1988) propone pensar las instituciones educativas no solamente como centros docentes, sino como centros de investigación donde la autonomía del educando se refleje en su capacidad de decidir sobre su propio proceso de enseñanza y aprendizaje, y en la posibilidad de enfrentarse a lo desconocido sin la constante corrección o dirección del docente, lo que promovería en el estudiante la necesidad de investigar, no solo de forma autónoma, sino también creativa. De ahí que en la ejecución, el rol del estudiante sea operativo y participativo. Este papel supone desarrollar la actividad de forma que él mismo pueda experimentar y elegir la forma en la que aprende mejor y, en consecuencia, pueda seleccionar lo que mejor se adapta a sus cualidades, es decir, que sea él mismo quien dirija su propio aprendizaje (Bray \& McClaskey, 2013). La selección puede estar orientada por aquellos materiales previamente elegidos por el docente en la fase de planificación. El trabajo por rincones en Educación Infantil es un ejemplo claro de esta forma de proceder (Morales, 2012).

Respecto al potencial creativo del ser humano y su desarrollo en la ejecución, Rodríguez (2012) hace referencia a la importancia de generar un contexto escolar donde cada estudiante experimente de forma práctica el contenido de estudio y se le ofrezca un espacio tanto físico como temporal para que se formule preguntas y las comparta con los compañeros. De esta manera las ideas cotidianas pueden cuestionarse y dar lugar a nuevas formas de entender y explicar el mundo. En este sentido, la intervención docente estaría destinada a que los alumnos puedan expresar y explorar sus propias ideas y ponerlas a prueba, es decir, a que puedan generar ideas maravillosas en palabras de Duckworth (1994).

Herrán (2012) sostiene que las producciones creativas no solo dependen de las características del sujeto creador sino también, en gran medida, de la respuesta o participación del contexto que en ocasiones ignora o sanciona la novedad, validez, pertinencia y utilidad del producto creado, lo cual abre una puerta a las características que debería tener el entorno escolar para 
promover la creatividad de cada alumno. El docente y el aula funcionan como contextos receptores activos que pueden facilitar, obstaculizar o impedir el proceso creativo y sus consecuencias. Elisondo et al. (2011) argumentan «que los espacios creativos en educación deberían promover mini-creatividades, es decir, comprensiones novedosas, originales y alternativas acerca de los objetos de conocimiento» (p. 148). Para favorecer el desarrollo de mini-creatividades en el aula proponen ofrecer actividades inesperadas, con consignas amplias y abiertas en las tareas que le permita a cada alumno llevar el control y tomar decisiones en cuanto a su propio aprendizaje.

Un ejemplo de personalización desde la singularidad lo encontramos en Tourón y Santiago (2015) quienes describen el llamado flipped classroom ( «aula al revés» o «aula invertida», en castellano) que cuestiona un modelo de escuela que pretende enseñar a la diversidad del alumnado los mismos contenidos, con el mismo nivel de profundidad y al mismo ritmo, dificultando así la atención de las necesidades y demandas de cada ser singular. Invertir el aula se perfila como una opción pedagógica que se centra en el aprender más que en el enseñar e incorpora, además del modelo expositivo de clase magistral, diversas opciones organizativas y pedagógicas aprovechándose de los medios y recursos digitales disponibles. Si en el modelo tradicional, los alumnos tienen escasa información sobre lo que se va a trabajar en clase y es el maestro el encargado de transmitirlo, en fipped classroom los estudiantes llegan al aula con preguntas e inquietudes concretas sobre el tema o problema en cuestión y el espacio-tiempo escolar se dedica a la práctica, a la investigación y a la aplicación del aprendizaje; así como la colaboración entre los estudiantes. Lo anterior supone que durante el tiempo de clase aumentan las interacciones alumno-profesor promoviendo el desarrollo de las potencialidades y el talento de todos y cada uno de los participantes (Tourón y Santiago, 2015). Una concreción la encontramos en un estudio que evidencia que la inclusión de contenido digital online como videos, tareas y enlaces adicionales que ayude a visualizar las matemáticas mejora el rendimiento académico de los estudiantes en esta disciplina (Fulton, 2012). En el aula invertida, la visualización de videos se lleva a cabo fuera del tiempo en el que están reunidos los estudiantes.

Uno de los muchos ejemplos de diseño de programa que pueden mostrarse en esta línea es el de Tourón, Marcos y Tourón (2010) en el que los autores aseguran que los alumnos de $4^{\circ}$ curso de Primaria han podido «experimentar un nivel de reto y satisfacción intelectual que de ordinario la clase regular no les proporciona, al ser el nivel de individualización mucho mayor» (p. 134) tras haberles aplicado un programa de enseńanza multimedia interactivo de Matemáticas. 


\subsection{La singularidad en la evaluación del aprendizaje}

En el momento de diseñar e implementar la actividad evaluativa consideramos relevante tener en cuenta la singularidad de la persona, destacando la función formativa de la evaluación donde se prima el proceso sobre el resultado, lo que revierte en que la evaluación se convierte en una ocasión más para aprender. La retroalimentación que se proporciona respecto a los aprendizajes, que ocurre dentro del propio proceso, generará mejores resultados, no solo por detectar los errores sino también por reconocer los aciertos (Fonseca, 2009; Garello \& Rinaudo, 2013).

Para conocer si en una acción formativa se ha conseguido lo que se pretendía es necesario evaluarla. En este sentido, nos centramos en la evaluación del aprendizaje y en la evaluación para el aprendizaje. Son numerosos los trabajos que abogan por la evaluación formativa (por ejemplo: Taras, 2005; LópezPastor, Pintor, Muros \& Webb, 2013). Denominamos evaluación formativa, como actividad humana que es, a aquella que le permite al estudiante aprender de lo que ha realizado y de la propia evaluación (Casanova, 2012); para que esto ocurra, es necesario detectar los errores y los aciertos, tener conciencia de ambos y poner en marcha acciones para evitar que vuelvan a ocurrir los primeros y potenciar los segundos. En la detección cabrían varias posibilidades, dos de ellas: 1) Tradicionalmente el profesor los detecta y comunica al estudiante; es lo que se denomina heteroevaluación y 2) Desde los conceptos de la metacognición, es el propio alumno quien detecta sus propios errores y aciertos. En ambos casos es necesario que el estudiante se haga consciente de ello. Por tanto, es posible que la segunda forma de proceder le permita caer en la cuenta con mayor significación; de modo que la puesta en marcha de las acciones necesarias ayude al aprendizaje permanente (Hattie \& Timperley, 2007) y a la mejora de la calidad de la educación (Pérez Juste, 2001).

Lo que hemos apuntado hasta aquí hace que sea necesario, en todo proceso formativo, proveer un espacio seguro para dar al estudiante la posibilidad de equivocarse sin que ello le traiga consecuencias negativas, un contexto donde se pueda probar, errar y compartir experiencias, donde se pueda investigar con libertad y sin miedo al error (Rodríguez, 2012). Consideramos este enfoque muy relevante para el personal desarrollo de cada estudiante ya que, entre el fomento de otras potencialidades, se entiende que la capacidad autorreguladora, propia del aprendizaje metacognitivo, lleva al conocimiento (Bernardo, 2011a y 2011b). De este modo, la evaluación que hace el profesor cumple con un papel fundamental para proporcionar al estudiante pautas que le permitan autoevaluarse y corregir sus propios fallos, retomar aquello que no haya 
comprendido o profundizar en lo que le parece más atractivo. Cada persona, por su ser único, irá desarrollando el propio perfeccionamiento en función de sus propias necesidades e intereses, lo que significa que es el proceso de enseñanza-aprendizaje el que ha de adaptarse a esta singularidad de cada persona y no al contrario, como hemos apuntado previamente.

Por otro lado, es necesario que el docente conozca con mayor profundidad lo que implica la evaluación para el desarrollo de competencias que se hayan evidenciado a lo largo de la etapa formativa. El docente deberá, por tanto, diseńar el proceso de enseńanza-aprendizaje de modo que proporcione al alumno diversas ocasiones para demostrar que las ha conseguido y se manifiesten en los resultados de aprendizaje esperados. Las pautas de Rodríguez, Roca, de Amo, Alias y Márquez (2011) serían útiles para la formación docente en estos aspectos. Desde la singularidad de cada persona, para evaluar, será necesario proporcionar un abanico de herramientas que agrupen los distintos tipos de enseñanza para conocer y utilizar diversas formas de evaluación. Si los profesores, por lo general, no han recibido formación específica sobre evaluación; es posible que desconozcan aquellos tipos de pruebas que no hayan utilizado a lo largo de su propio proceso formativo, previo al desempeño de la labor docente y, en consecuencia, el abanico de posibles herramientas de evaluación se ve limitado. Un estudio realizado por Arteaga, Bujalance y Perochena (2014) precisamente expone pautas en materia de formación docente que identifican la singularidad del estudiante como punto neurálgico en materia de evaluación.

Dentro de los procesos formativos, conviene reflexionar y poner en marcha acciones concretas para que la evaluación se convierta en una ocasión para aumentar la motivación del alumno hacia su propio aprendizaje (Fonseca, 2009). Puesto que todo aprendizaje requiere un esfuerzo, la labor docente radica en orientar los esfuerzos del estudiante hacia la consecución de los objetivos (Bernardo \& Basterretche, 2004), hacia su perfección en tanto que persona. La evaluación puede, en este sentido, convertirse en un elemento de motivación si se lleva a cabo desde la realidad de quien es cada estudiante y desde sus propias capacidades y potencialidades (Bernardo \& Javaloyes, 2015). Por ello, partir de una evaluación diagnóstica que permita sentar las bases de la actividad educativa será la clave para proporcionar una satisfacción al evidenciar los aprendizajes adquiridos y, por tanto, se convertirá en un elemento de motivación para continuar aprendiendo.

Si las acciones evaluadoras se conciben como un momento relevante de encuentro interpersonal profesor-alumno para la formación integral de este, a través de ellas puede ocurrir un perfeccionamiento de sus facultades 
específicamente humanas. Las acciones evaluadoras pueden ser un ámbito de relación entre el docente y el estudiante. Entender la corrección de las pruebas de evaluación, la lectura de un trabajo del alumno, escuchar su exposición, el momento de revisión de un examen, etc. como intercambios generosos de capacidades nos permitirá descubrir un valor mucho más profundo de la evaluación que el mero hecho de otorgar una calificación. Este valioso intercambio, plenamente formativo, nos da la posibilidad de trascender unas fronteras donde los dos — docente y estudiante — se enriquecen mutuamente en un nivel creativo específicamente humano.

\section{Consideraciones Finales}

A la vista de las reflexiones anteriores consideramos relevante crear en el aula un clima que fomente el desarrollo del discernimiento de la información importante, o fiable, de la que no lo es. La actividad escolar debe promover la conciencia de identidad personal ayudando a descubrir el gran valor de todo ser humano. En esta era digital se ha de poner particular empeño en ayudar a los estudiantes a descubrir y ser críticos con las posibles falsas identidades digitales y actuar en consecuencia.

Por otro lado, hemos mostrado la necesidad de que todo proceso educativo tenga muy en cuenta la singularidad de cada persona como una de sus dimensiones esenciales. Hemos revisado los procesos de planificación, ejecución y evaluación y, a la vista de todo ello, aportamos algunos criterios aplicables a la hora de diseñar y ejecutar dichas fases desde la singularidad de cada estudiante considerándolas como diferentes «lecturas» de un mismo proceso en el que cada una de ellas se debe orientar a las otras dos.

La coherencia entre objetivos, recursos y evaluación debe estar garantizada. Para conseguir niveles aceptables de eficacia y eficiencia, al redactar los objetivos, contenidos y competencias de un determinado curso es necesario explicitar tanto los modos en que se va a evaluar su logro como los recursos necesarios para realizar las actividades con sentido pedagógico. En particular, destacamos la importancia de la evaluación formativa que, al detectar logros o fallos en el desempeńo de las tareas, nos permite en el momento más próximo a la ejecución platearnos una continua remodelación de los objetivos y recursos lo que supondría un aprendizaje más significativo y en consecuencia, más motivador.

Las consideraciones anteriores nos llevan a proponer los siguientes criterios: 
Criterios para una planificación singularizadora:

1. Las actividades formativas deben diseñarse de forma que haya suficientes momentos para que los alumnos, una vez alcanzados los objetivos comunes, mínimos, puedan trabajar en el logro de objetivos individuales acordes con sus capacidades o intereses.

2. En la organización del tiempo diario conviene incluir momentos en que el alumno pueda decidir reflexivamente sobre las tareas y actividades que, en función de sus circunstancias concretas y de su posible proyecto personal, puede/debe realizar.

3. La agrupación de alumnos, dentro del aula, debe preverse de forma que permita la flexibilidad necesaria para que puedan realizar actividades individuales o cooperativas.

4. La planificación del uso de los materiales didácticos y las estrategias de enseñanza-aprendizaje debe permitir el progreso de cada alumno de acuerdo con sus posibilidades de modo que no se frene su desarrollo.

Criterios para una ejecución singularizadora:

5. El desarrollo de las clases y las actividades en general deben tener en cuenta la evolución de las circunstancias personales de cada alumno y adecuarse a las necesidades que, a lo largo del curso, van surgiendo como fruto de los avances reales conseguidos o de otras circunstancias incluso del mundo social o del entorno escolar.

6. Puesto que el estudiante no es un mero receptor de información, es importante contar con su percepción sobre la cantidad, calidad y modos de ejecución de los procesos de enseñanza-aprendizaje. Escuchar y tener en cuenta las aportaciones de los estudiantes es un factor relevante en su proceso de crecimiento personal.

7. Recomendamos implementar la experimentación, el autoaprendizaje y el uso de metodologías inductivas así como la utilización estratégica del error como herramienta de aprendizaje.

Criterios para una evaluación singularizadora:

8. Es importante reconocer que existen distintas funciones de la evaluación: certificadora, clasificadora, diagnóstica y formativa, entre otras. Desde la singularidad de cada estudiante hacemos especial hincapié en el uso de las dos últimas, fomentando así mismo la autoevaluación. 
9. La evaluación debe ser contemplada como elemento esencial en la planificación, ya que esta debe incluir la identificación de la forma de evidenciar que los objetivos han sido alcanzados.

A modo de prospectiva para futuros trabajos consideramos relevante estudiar la forma de ayudar a cada alumno a que sea consciente de que su vida y su pensamiento no son un simple resultado de acciones ajenas, fomentando la responsabilidad personal en su desarrollo, lo cual exige hacer aportaciones, únicas y singulares, a su entorno familiar, escolar, social, etc.

\section{REFERENCIAS BIBLIOGRÁFICAS}

Area, M. \& Ortíz, M. (2000). Medios de comunicación, interculturalismo y educación. Comunicar, 15, 114-122.

Artamendi, J. A. (2007). Hablando con Xabier Zubiri II. Sobre el convivir como problema. Desde «Sobre el hombre». Lasarte-Oria: Etor-Ostoa.

Arteaga, B., Bujalance, L. \& Perochena, P. (2014). Un acercamiento a la opinión de los profesores sobre el proceso de evaluación en la universidad online. Revista Internacional de Educación y Aprendizaje, 2(1), 49-55.

Bernal, A. (2012). Educar en la libertad y la responsabilidad. Una tarea compleja. Edetania: estudios y propuestas socio-educativas, 42, 75-90.

Bernal, A. (2014). Competencia emprendedora e identidad personal. Una investigación exploratoria con estudiantes de Educación Secundaria Obligatoria. Revista de Educación, 363, 384-411. DOI: 10-4438/1988-592X-RE-2012-363-192.

Bernardo, J. (coord). (2007). Cómo personalizar la educación. Una opción de futuro. Madrid: Narcea.

Bernardo, J. (2011a). Enseñar hoy. Una didáctica básica para profesores. Madrid: Síntesis.

Bernardo, J. (coord.) (2011b). Educación personalizada: principios, técnicas, y recursos. Madrid: Síntesis.

Bernardo, J. \& Basterretche, B. (2004). Técnicas y recursos para motivar a los alumnos. Sexta ed. Madrid: Rialp.

Bernardo, J. \& Javaloyes, J. J. (2015). Motivar para educar. Ideas para educadores: docentes y familias. Madrid: Narcea.

Bravo, J. L. (2004). Los medios de enseñanza clasificación, selección y aplicación. Pixel-Bit: Revista de medios y educación, 24, 113-124.

Bray, B. \& McClaskey, K. (2013). A step-by-step guide to personalize learning. Learning \& Leading with Technology, 40(7), 12-19. 
Burgos, J. M. (2007). La filosofía personalista de Karol Wojtyla. Madrid: Palabra.

Calderero, J. F., Aguirre, A. M., Castellanos, A., Peris, R. \& Perochena, P. (2014). Una nueva aproximación al concepto de educación personalizada y su relación con las TIC. Teoría de la educación. Educación y cultura en la sociedad de la información, 15(2). Recuperado de http://campus.usal.es/ -revistas_ trabajo/index.php/revistatesi/ article/view/11890

Campos, R. (2008). Incertidumbre y complejidad: reflexiones acerca de los retos y dilemas de la pedagogía contemporánea. Actualidades Investigativas en Educación, 8(1). Recuperado de http://www.redalyc.org/ pdf/447/44780102.pdf. https://doi.org/10.15517/aie.v8i1.9326.

Casanova, M. A. (2012). Evaluación de competencias básicas. Madrid: Muralla.

Castells, M. (1997). La era de la información. Economía, sociedad y cultura. Vol. 1 La sociedad red. Madrid: Alianza.

De Bono, E. (2012). Seis sombreros para pensar (30 imp.). Barcelona: Paidós.

Duckworth, E. (1994). Cómo tener ideas maravillosas. Y otros ensayos sobre cómo enseñar y aprender. Madrid: Antonio Machado.

Elisondo, R., Rinaudo, M. \& Donolo, D. (2011). Actividades inesperadas como oportunidades para la creatividad. Contextos creativos en la educación superior Argentina. Innovación Educativa, 11(57), 147-156. Recuperado de http://www.redalyc.org/articulo.oa?id=179422350016

Escotet, M. (2002). Desafíos de la educación superior en el siglo de la incertidumbre. Cuaderno de Investigación en la Educación, 18. Recuperado de http://cie.uprrp.edu/cuaderno/ediciones/18/pdfcuaderno18/c18art1.pdf

Fonseca, H. (2009). Retroalimentación durante el proceso de enseñanza-aprendizaje: un arma de doble filo. Salud, arte y ciudadano, 2, 50-52.

Fulton, K. (2012). Upside down and inside out: Flip your classroom to improve student learning. Learning \& Leading with Technology, 39(8), 12-17.

García, A. (2013). Proponiendo un concepto nuclear latente en educación: las Necesidades Educativas Personales (NEP). (Tesis doctoral). Universidad Autónoma de Madrid. Madrid.

García Hoz, V. (1988). Educación personalizada. Madrid: Rialp.

García Hoz, V. (1993). Introducción general a una pedagogía de la persona. Madrid: Rialp.

Garello, M. V. \& Rinaudo, M. C. (2013). Autorregulación del aprendizaje, feedback y transferencia de conocimiento. Investigación de diseño con estudiantes universitarios. Revista Electrónica de Investigación Educativa, 15(2), 131-147. Recuperado de http://redie.uabc.mx/vol15no2/contenido-garellorinaudo.html 
Gros, B. (2009, junio). De consumidores de información a productores de conocimiento. El aprendizaje colaborativo en la red. Ponencia presentada en VIII Jornadas de Buenas Prácticas Educativas, Zaragoza, España.

Hattie, J. \& Timperley, H. (2007). The Power of Feedback. Review of Educational Research, 77(1), 81-112. https://doi.org/10.3102/003465430298487

Herrán, A. de la (2012). Análisis relativo del proceso y el producto creativo. En D. Velázquez Vázquez (Coord.), Calidad y creatividad aplicada a la enseñanza superior (pp. 301-334). México: Porrúa-Universidad Nacional Autónoma de México.

Jenkins, J. M. \& Keefe, J. W. (2001). Strategies for Personalizing Instruction: A Typology for Improving Teaching and Learning. NASSP Bulletin, 85(629), 72-85. https://doi.org/10.1177/019263650108562908

Jouve, N. (2013). La genética y la dignidad del ser humano. Cuadernos de Bioética, 24(80), 91-100.

López-Pastor, V. M., Pintor, P., Muros, B. \& Webb, G. (2013). Formative assessment strategies and their effect on student performance and on student and tutor workload: the results of research projects undertaken in preparation for greater convergence of universities in Spain within the European Higher Education Area (EHEA). Journal of Further and Higher Education, 37(2), 163-180. https://doi.org/10.1080/0309877X.2011.644780

Maritain, J. (1947). Distinguir para unir, los grados del saber. Buenos Aires: Desclée de Brouwer.

Marquès, P. (2000). Los medios didácticos y los recursos educativos. Revista DIM. Recuperado de https://dl.dropboxusercontent.com/u/20875810/dim/ revistaDIM31/revistanew.htm

Martín, X., Novella, A. \& Puig, J. (2013). Citizenship without certainties. Encounters in Theory and History of Education, 14, 99-116. https://doi. org/10.15572/enco2013.07

Meléndez, M. C. (2014). «\#Socorro! ¿Qué hace mi hijo en las redes sociales?» Albacete: UNO Editorial.

Morales, F. M. (2012). Intervención psicoeducativa en centros de educación infantil de primer ciclo. Revista Iberoamericana para la Investigación y el Desarrollo Educativo, 9.

Palacios, L. E., Medina, R., Forment, E., Román, M., Moreno, P., Marín, R. et al. (1989). El concepto de persona. Madrid: Rialp.

Pariente, J. L. \& Perochena, P. (2013). Didáctica de la educación en valores en la ESO. Una propuesta utilizando las tecnologías para el aprendizaje y el conocimiento. Pixel-Bit, Revista de Medios y Educación, 42, 195-208. 
Paul, M. \& Stroh, D. P. (2006). Managing your time as a leader. Reflections, 7(4), $1-16$.

Pérez Juste, R. (2001). Calidad de la educación: personalización educativa y pertinencia social. Recuperado de http://www.uv.es/soespe/Paz-PerezJuste.htm

Rendón, M. (2003). Propuesta pedagógica para el desarrollo de la creatividad en niños en edad de preescolar. Material no publicado. La Habana: Instituto Central de Ciencias Pedagógicas.

Rodríguez, M. (2012). Mitos: el mérito de saber equivocarse. Padres y Maestros, 344, 27-30.

Rodríguez, D., Roca, J., de Amo, J. M., Alias, A. \& Márquez, J. (2011). Formación del profesorado universitario para el diseño, el desarrollo y la evaluación de competencias. Educar, 47, 381-401.

Serrano, J. (2013). La expresión de la identidad en la sociedad digital: una aproximación teórica a las redes sociales. En B. Lloves y F. Segado (coords.), I Congreso Internacional de Comunicación y Sociedad Digital. Logrońo: Universidad Internacional de La Rioja.

Taras, M. (2005). Assessment-summative and formative-some theoretical reflections. British Journal of Educational Studies, 53(4), 466-478. https://doi. org/10.1111/j.1467-8527.2005.00307.x

Tonucci, F. (2008, 29 de diciembre). La misión principal de la escuela ya no es enseñar cosas. La Nación.

Tourón, J. \& Santiago, R. (2015). El modelo Flipped Learning y el desarrollo del talento en la escuela. Revista de Educación, 368, 196-231.

Tourón, J., Marcos, G. \& Tourón, M. (2010). La educación online con alumnos de alta capacidad intelectual. Evaluación de una intervención en el ámbito de las Matemáticas. REIFOP, 13(1). Recuperado el 15-06-2016 de http://sid.usal.es/idocs/F8/ART15557/educacion_online_alumnos_alta_ capacidad.pdf

Vila, I. \& Casares, R. (2009). Educación y sociedad. Barcelona: Horsori.

Velazco, M. (2015). El alumno como persona, protagonista de la educación. En J. F. Calderero (dir.), Seminario Internacional de Educación Personalizada (SIEP). Conferencia trasmitida on-line. Recuperada el 15-06-2016 de http://tv.unir.net/videos/15262/368/1330/1832/0/El-alumno-comopersona-protagonista-de-la-educacion

Wisker, G., Exley, K., Antoniou, M. \& Ridley, P. (2012). Trabajando individualmente con cada estudiante: Tutoría personalizada, coaching, mentoría y supervisión en Educación Superior. Madrid: Narcea. 